\title{
Variations in Ribosomal RNA Gene Loci in Spider Lily (Lycoris spp.)
}

\author{
Yu-Chu Chang and Chou-Tou Shii ${ }^{1}$ \\ Department of Horticulture, National Taiwan University, Taipei, Taiwan, ROC \\ Mei-Chu Chung ${ }^{1}$ \\ Institute of Plant and Microbial Biology, Academia Sinica, No. 128, Sec. 2, Academia Road, Taipei, \\ 11529, Taiwan, ROC
}

\begin{abstract}
Additional INDEX words. FISH, 45S rDNA, 5S rDNA, DAPI-positive band, karyotype, colocalization, interspecific hybrid
Abstract. Lycoris species of the Amaryllidaceae are important ornamental and medicinal plants in Asia. Karyotypes of Lycoris species have been studied extensively since the time when their chromosome numbers were first counted over 80 years ago. Based on karyotype, Lycoris taxa can be classified into the monomorphic A group, dimorphic MT group, and the sterile dikaryotype MT-A group. Numerous reports dealing with karyotype analysis and phylogenetic relationship in the genus Lycoris have been published. However, there are disputes and controversies regarding karyotype evolution resulting from lacking efficient and reliable markers for chromosome identification in the genus Lycoris. In this study, we applied fluorescent in situ hybridization (FISH) to visualize the 5S and 45S rDNA loci on chromosomes as landmarks for chromosome identification in Lycoris taxa. In total, 12 accessions of three karyotype groups, including nine species and three artificial dikaryotype hybrids, were investigated. A high degree of variation in the number and position of 5S and 45S rDNA loci was detected among Lycoris taxa. There were four to 14 FISH signals of $5 \mathrm{~S}$ rDNAs and two to 12 FISH signals of $45 \mathrm{~S}$ rDNAs observed in each investigated Lycoris accession. Lycoris accessions with the same karyotype $2 n=22 \mathrm{~A}$ may have different numbers of rDNA loci, which distributed at different chromosomal positions. In an interspecific hybrid, the number and chromosomal position of both $5 \mathrm{~S}$ and $45 \mathrm{~S}$ rDNA loci were either the combinations of those in their parental species or considerably modified. Overlapping FISH signals of $5 \mathrm{~S}$ and $45 \mathrm{~S}$ rDNAs were colocalized with a 4', 6-diamidino-2-phenylindole-positive band at the end of the p-arm on almost every T-type chromosome (but not the A-type chromosomes). Based on the features of T-type chromosomes, the possibility of centromeric fission in karyotypic evolution of Lycoris is discussed.
\end{abstract}

The genus Lycoris (Amaryllidaceae) comprises $\approx 30$ taxa (Kurita and Hsu, 1998), most of which have been grown for centuries as ornamentals and medicinal plants in China, Japan, and Korea. Lycoris taxa vary in flower shape and color as well as chromosome number and karyotypes, posing a considerable challenge in studying their phylogenetic relationships (Kurita and Hsu, 1998). In natural habitats, interspecific hybridization is frequent and has been proven to be an important mode of speciation in the genus Lycoris (Kurita, 1988; Kurita and Hsu, 1996). The interspecific hybrids can be propagated asexually through bulblets, and some of them have become widely dispersed. Several chromosome variations have been found in 38 populations of Lycoris sanguinea (Kurita, 1989). The heterozygous nature of Lycoris taxa was also shown in five diploid progenitor species in which genetic segregation of several allozyme loci was observed (Ma et al., 2004). On the other hand, a Lycoris taxon may show a genetic constancy among widely distributed populations (Kurita and Hsu, 1998). A completely sterile triploid Lycoris radiata var. radiata $(2 n=$ $33 \mathrm{~A}$ ), which is widely dispersed in Japan and Korea, shares the

Received for publication 11 Aug. 2009. Accepted for publication 5 Oct. 2009. This study was supported by grants from the Council of Agriculture of Taiwan to Chou-Tou Shii and from Academia Sinica, Taiwan, to Mei-Chu Chung. We sincerely thank Yu-Ming Ju (Institute of Plant and Microbial Biology, Academia Sinca, Taipei, Taiwan) and Fure-Chyi Chen (National Pingtung University of Science and Technology, Pintung, Taiwan) for critical reading of this manuscript. Special acknowledgements are given to anonymous reviewers for their useful suggestions to earlier versions of the manuscript.

1Corresponding authors. E-mail: shiict@ntu.edu.tw; bomchung@gate.sinica. edu.tw. same karyotype (Kurita, 1987b), allozyme loci (Chung, 1999), and sequences of two genes: the lectin gene in the nuclear genome and the maturase gene in the chloroplast genome (Hayashi et al., 2005). These populations could have been derived from only one or a few bulblets originally introduced from China $\approx 3000$ years ago.

Nishiyama (1928) was the first to count chromosome numbers of Lycoris species and gave $n=11$. A large number of cytogenetic studies of Lycoris have been published since then (review by Jones, 1998). Lycoris taxa are classified into three groups based on chromosome complements: acrocentrics (A-type), metacentrics (M-type), and telocentrics (T-type) (Kurita, 1986). Plants with an A or MT karyotype are fertile diploids, whereas those with an MT-A karyotype are sterile (Kurita and Hsu, 1998). Recently, numerous investigations of Lycoris taxa were focused on cytological analyses, taxon identifications, and molecular phylogenetic relationships (Hori et al., 2006; Hsu et al., 1994; Kim and Lee, 1991; Kurita, 1987a, 1987b, 1988; Lee and Kim, 1987; Liu and Hsu, 1989; Shi et al., 2006; Yuan et al., 1998). However, it remains difficult to differentiate species with the same karyotype or to discriminate T-type from A-type chromosomes in some MT-A dikaryotype hybrids (Kurita, 1986). Furthermore, little knowledge on the ancestral karyotype as well as the mechanism of chromosome evolution in Lycoris species is available (Inariyama, 1951; Kurita, 1988). To address these questions, efficient and reliable landmarks for chromosome or even for chromosomal arm identification are urgently needed.

Ribosomal RNA genes (rDNAs) have been proven to be reliable landmarks in karyotype studies in Aegilops (Badaeva 
et al., 1996; Castilho and Heslop-Harrison, 1995), Arabidopsis (Fransz et al., 1998), Hordeum (Taketa et al., 1999), and Trifolium (Ansari et al., 1999). Karyotyping with rDNA loci can reflect the phylogenetic relationship among closely related species. In higher eukaryotes, rDNAs are organized into two distinct multigene families, one coding for $45 \mathrm{~S}$ rRNA and the other coding for $5 \mathrm{~S}$ rRNA (Suzuki et al., 1996). In plants, $45 \mathrm{~S}$ rDNAs are highly repeated and arranged in tandem at one or a few chromosomal loci. A chromosome locus where $45 \mathrm{~S}$ rDNAs are active in transcription is often associated with a nucleolus. This locus is called a nucleolar organizing region (McClintock, 1934). A 5S rDNA locus also contains a tandem array of hundreds or even thousands of repeats, which is usually located separately from $45 \mathrm{~S}$ rDNA locus (review by Drouin and Moniz de Sa, 1995). The number and chromosomal position of 5S rDNA loci as well as those of $45 \mathrm{~S}$ rDNA loci may vary among closely related plant species (references in Chung et al., 2008) [e.g., Phaseolus vulgaris (Pedrosa-Harand et al., 2006) and certain Oryza species (Chung et al., 2008)].

The fluorescent in situ hybridization (FISH) technique allows physical mapping of rDNA locus regardless of chromosomal feature and transcriptional activities of rDNA repeats (Cabrero and Camacho, 2008; Chung et al., 1993, 2008; LindeLaursen et al., 1992). The locations of $45 \mathrm{~S}$ rDNA loci have been determined by FISH for Lycoris chinensis var. sinuolata $(2 n=$ $16,6 \mathrm{M}+2 \mathrm{SM}+8 \mathrm{~T}$ ) and some Korean endemic Lycoris species (Lee and Kim, 2000, 2004). The results of 5S and 45S rDNA FISH analyses suggested that $L$. radiata var. radiata $(2 n=3 \mathrm{x}=$ 33 ) was derived from a diploid botanical variety, $L$. radiata var. pumila $[2 n=22 \mathrm{~A}$ (Hayashi et al., 2005)]. Also, rDNA loci were used as reliable landmarks for checking the formation of unreduced gametes of three interspecific hybrids and a selfed progeny of Lycoris sprengeri (Ogawa et al., 2006).

In this study, we applied rDNA FISH to investigate the variations of $5 \mathrm{~S}$ and $45 \mathrm{~S}$ rDNA loci on chromosomes of 12 Lycoris taxa, including nine species and three artificial hybrids. Distributions of rDNA loci on chromosomes of most taxa are reported for the first time except for L. radiata var. pumila and
L. sprengeri (Ogawa et al., 2006). We showed that the number and locations of $5 \mathrm{~S}$ and $45 \mathrm{~S}$ rDNA loci varied among taxa of three karyotype groups and even among taxa within a karyotype group. This study is also the first to observe $5 \mathrm{~S} \mathrm{rDNA}$ loci were colocalized with $45 \mathrm{~S}$ rDNA loci at the end of p-arm of T-type chromosomes in Lycoris taxa.

\section{Materials and Methods}

Plant materials. Lycoris accessions used in this study are listed in Table 1. Lycoris radiata var. pumila, $L$. sprengeri, $L$. rosea, L. haywardii, L. chinensis, L. Xhoudyshelii, and two artificial hybrids with a MT-A karyotype were introduced from China, whereas L. albiflora was from Japan. Accessions of native golden spider lily ( $L$. aurea, $2 n=14,8 \mathrm{M}+6 \mathrm{~T}$ ) were either collected from natural habitats in northern Taiwan or obtained from a local grower. A variant of $L$. radiata var. pumila $(2 n=22,22 \mathrm{~A})$ and a curly leaved variant of $L$. sprengeri $(2 n=22=22 \mathrm{~A}$, accession LSM) were collected from Ma-Tzu, an island close to eastern China. All plants were grown in pots or flats using a soilless medium supplied with slow-release fertilizers and maintained in the greenhouse at the experimental farm of the Department of Horticulture, National Taiwan University, Taipei, Taiwan. At least three plants of each accession were sampled for further studies.

Chromosome PREPARATION. Young roots $\approx 0.5 \mathrm{~cm}$ long were collected at $1200 \mathrm{HR}$, pretreated with $2 \mathrm{~mm} 8$-hydroxyquinoline at $18{ }^{\circ} \mathrm{C}$ for $4 \mathrm{~h}$, then fixed in Farmer's fixative [95\% ethanol + glacial acetic acid $(3: 1 \mathrm{v} / \mathrm{v})$ ] overnight at room temperature, and were stored at $-20{ }^{\circ} \mathrm{C}$ until used. The fixed root tips had been washed with distilled water before they were macerated in an enzyme mixture containing 6\% pectinase (Sigma Chemical, St. Louis, MO) and 6\% cellulase (Onozuka R-10; Yakult Honsha, Tokyo, Japan) in $75 \mathrm{~mm} \mathrm{KCl}(\mathrm{pH}=4.0)$ at $37^{\circ} \mathrm{C}$ for $75 \mathrm{~min}$. After having been washed with distilled water, the softened tissues were squashed on a slide with a drop of fixative. Slides were air-dried and then stored at $-80{ }^{\circ} \mathrm{C}$ until used for FISH analyses.

Table 1. Chromosome complements of Lycoris taxa used in this study and the number and positions of rDNA fluorescent in situ hybridization (FISH) signals.

\begin{tabular}{|c|c|c|c|c|c|c|c|c|c|}
\hline \multirow[b]{2}{*}{ Lycoris accessions (origin) } & \multirow[b]{2}{*}{ Karyotypes $(2 n)$} & \multicolumn{4}{|c|}{ 45S rDNA FISH signals (no.) } & \multicolumn{4}{|c|}{ 5S rDNA FISH signals (no.) } \\
\hline & & Total & $\mathrm{M}^{\mathrm{z}}$ & $\mathrm{T}^{\mathrm{z}}$ & $\overline{\mathrm{A}^{\mathrm{z}}}$ & Total & M & $\mathrm{T}$ & $\mathrm{A}$ \\
\hline L. aurea & $14=8 \mathrm{M}+6 \mathrm{~T}$ & 6 & $-^{\mathrm{y}}$ & 6 & - & 12 & 6 & 6 & - \\
\hline L. chinensis & $16=6 \mathrm{M}+10 \mathrm{~T}$ & 10 & - & 10 & - & 12 & 1 & 11 & - \\
\hline L. radiata var. pumila ${ }^{\mathrm{w}}$ & $22=22 \mathrm{~A}$ & 4 & - & - & 4 & 4 & - & - & 4 \\
\hline L. radiata var. pumila (variant, Ma-Tzu) & $22=22 \mathrm{~A}$ & 3 & - & - & 3 & 4 & - & - & 4 \\
\hline L. haywardii & $22=22 \mathrm{~A}$ & 3 & - & - & 3 & 4 & - & - & 4 \\
\hline L. albiflora ${ }^{\mathrm{v}}$ & $17=5 \mathrm{M}+1 \mathrm{~T}+11 \mathrm{~A}$ & 4 & 1 & 1 & 2 & 6 & 2 & 1 & 3 \\
\hline L. $\times$ houdyshelii ${ }^{\mathrm{u}}$ & $30=3 \mathrm{M}+5 \mathrm{~T}+22 \mathrm{~A}$ & 12 & 1 & 5 & 6 & 14 & 1 & 6 & 7 \\
\hline L. aurea $\times$ L. radiata var. pumila & $18=4 \mathrm{M}+3 \mathrm{~T}+11 \mathrm{~A}$ & 4 & - & 3 & 1 & $8-9$ & $3-4$ & 3 & 2 \\
\hline L. chinensis $\times$ L. rosea & $19=3 \mathrm{M}+5 \mathrm{~T}+11 \mathrm{~A}$ & 6 & - & 5 & 1 & 7 & - & 5 & 2 \\
\hline L. longituba $\times$ L. radiata var. pumila & $19=3 \mathrm{M}+5 \mathrm{~T}+11 \mathrm{~A}$ & 9 & 2 & 5 & 2 & 10 & 2 & 6 & 2 \\
\hline
\end{tabular}

${ }^{\mathrm{z}} \mathrm{M}=$ metacentric; $\mathrm{T}=$ telocentric; $\mathrm{A}=$ acrocentric.

yot present.

${ }^{x}$ Two 45S rDNA loci were detected on A-type chromosomes by Ogawa et al. (2006).

${ }^{\text {w}}$ Four 45S rDNA loci were detected on A-type chromosomes by Ogawa et al. (2006).

${ }^{\mathrm{v}}$ L. albiflora $=$ L. traubii $(n=6,5 \mathrm{M}+1 \mathrm{~T}) \times L$. radiata $(n=11 \mathrm{~A})($ Kurita, 1987a).

${ }^{\mathrm{u}}$ Lycoris $\times$ houdyshelii $=$ L. longituba $(n=8,3 \mathrm{M}+5 \mathrm{~T}) \times$ unknown donor $(\mathrm{n}=11)($ Kurita, 1987a). 
FluORESCENCE IN SITU HYBRIDIZATION. FISH was performed according to the protocol of Chung et al. (2008). Plasmid DNA of the construct $\mathrm{p} T A 71$ with the entire coding region of the $45 \mathrm{~S}$ rRNA gene $(\approx 9 \mathrm{~kb})$ of Triticum aestivum (Gerlach and Bedbrook, 1979) and plasmid DNA of the construct pTA794 with the $5 \mathrm{~S}$ rDNA repeat unit (410 bp) of T. aestivum (Gerlach and Dyer, 1980) were labeled with biotin-16-dUTP and digoxigenin-11-dUTP, respectively, by nick translation (Roche Diagnostics, Penzberg, Germany) and used as probes for rDNA FISH analyses. The hybridization position of the biotinylated probe was immunologically detected using fluorescein isothiocyanate-conjugated avidin as the green signal (Vector Laboratories, Burlingame, CA), whereas that of the digoxigenated probe was detected using rhodamine-conjugated antidigoxigenin antibodies as the red signal (Roche Diagnostics). Chromosomes were counterstained with 4', 6-diamidino2-phenylindole (DAPI) in Vectashield $\left(1.5 \mu \mathrm{g} \cdot \mathrm{mL}^{-1}\right.$; Vector Laboratories). Images were acquired and processed as previously described in Kao et al. (2006) and Chung et al. (2008). For each accession, at least five chromosome complements with good labeling signals were photographed and analyzed.

For the sake of convenience, chromosomes in each complement were arranged into $\mathrm{M}, \mathrm{T}$, and A groups. In each group, chromosomes were arranged according to their length in descending order. Chromosomes with the same number in different complements are not necessarily homologous. The short arm and the long arm of a chromosome are indicated as $\mathrm{p}$-arm (p) and q-arm (q), respectively, in accordance with the International System for Human Cytogenetic Nomenclature. For example, M3p represents the short arm of the third metacentric chromosome in a given complement.

\section{Results}

DAPI staining could differentiate T-type chromosomes from A-type chromosomes. An example is shown in Figure 1, in which a MT-A dikaryotype species, L. Xhoudyshelii $(2 n=$ $30,3 \mathrm{M}+5 \mathrm{~T}+22 \mathrm{~A})$, had DAPI-positive bands present near the distal end of the $\mathrm{p}$-arm of all $\mathrm{T}$ - type chromosomes but absent on the A-type chromosomes. Some of the M-type chromosomes showed positively DAPI-stained bands near the centromere.

The results of rDNA FISH, which are summarized in Table 1 and described in detail subsequently, indicate the variations in the number and distribution pattern of both 45S rDNA loci and 5S rDNA loci among Lycoris taxa as well as among accessions of a taxon. The number of FISH signals of $45 \mathrm{~S}$ rDNAs and $5 \mathrm{~S}$ rDNAs ranged from two to 12 and from two to 14 , respectively. Two signals on two homologs correspond to one locus in a diploid.

MT- KARYOTYPE. Lycoris aurea $(2 n=14,8 \mathrm{M}+6 \mathrm{~T})$ had six FISH signals of 45S rDNAs and 12 FISH signals of 5S rDNAs (Table 1; Fig. 2A-D). Each T-type chromosome had 45S and 5S rDNA FISH signals overlapped at the end of its p-arm (T1pT6p). The second pair of the M-type chromosomes, M3 and M4, had the remaining six 5S rDNA FISH signals; each chromosome had one at the subtelomeric region of the p-arm, one at the proximal region on the p-arm, and one at the distal end of the q-arm (Fig. 2D). The intensity of the two signals at the proximal regions were weaker than that of the others, suggesting less rDNA repeats present at these two loci.

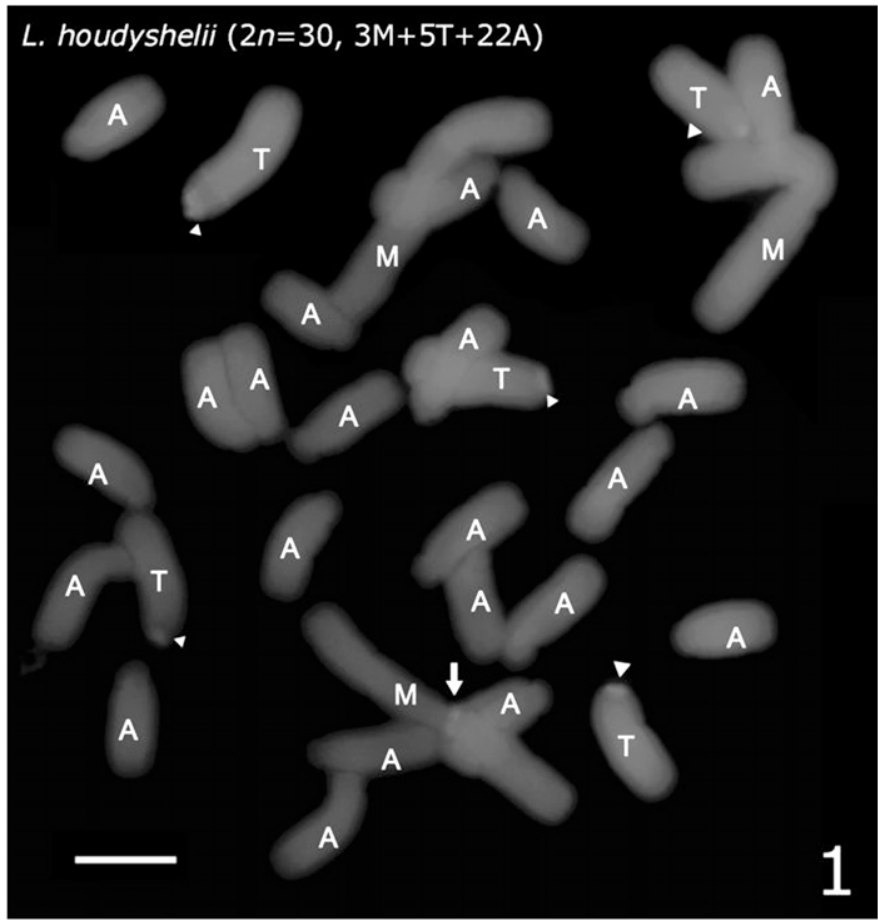

Fig. 1. Chromosome complement of Lycoris $\times$ houdyshelii $(2 n=30,3 \mathrm{M}+5 \mathrm{~T}+$ $22 \mathrm{~A}$ ) by $4^{\prime}$, 6-diamidino-2-phenylindole (DAPI) staining. There are three types of chromosomes: acrocentrics (A), metacentrics (M), and telocentrics (T). DAPI-positive bands present at the end of p-arm of T-type chromosomes (arrowheads) and near the centromere of M-type chromosomes (arrow); scale bar $=10 \mu \mathrm{m}$.

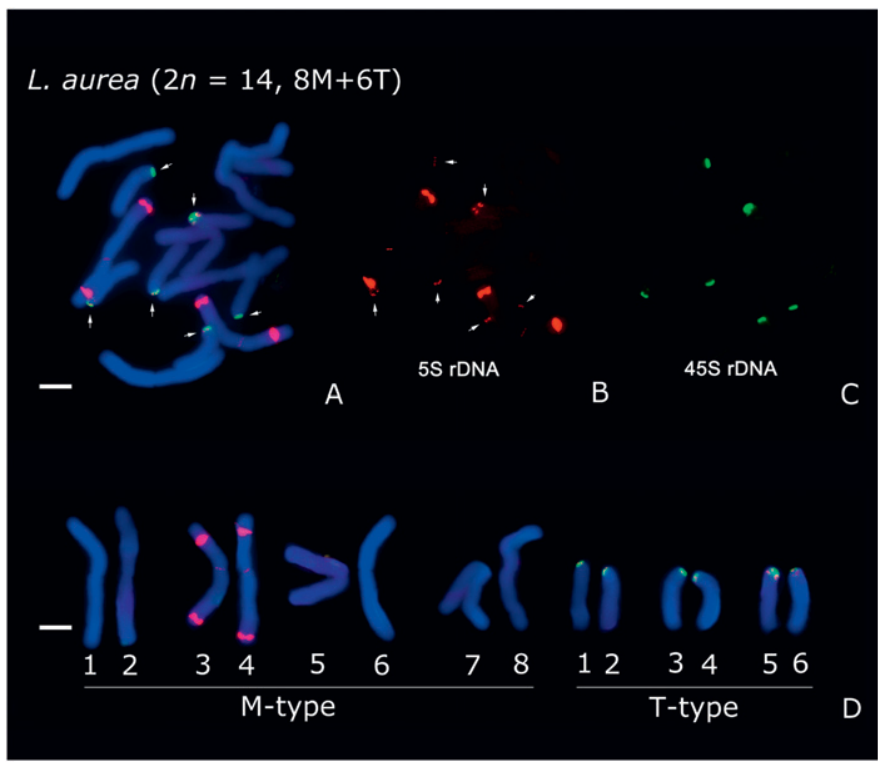

Fig. 2. rDNA fluorescent in situ hybridization (FISH) signals on the chromosome complement of Lycoris aurea $(2 n=14,8 \mathrm{M}+6 \mathrm{~T})$. (A) Merged image of 5S rDNA FISH signals (red) and 45S rDNA FISH signals (green) on chromosomes (blue). Arrows indicate the positions with overlapping rDNA FISH signals. (B) The distribution of 5S rDNA FISH signals in red. Arrows indicate the positions of signals overlapped with $45 \mathrm{~S}$ rDNA FISH signals shown in C. (C) The distribution of $45 \mathrm{~S}$ rDNA FISH signals in green. (D) Chromosome complements in $\mathbf{A}$ were arranged according to their length in descending order; scale bar $=10 \mu \mathrm{m}$. 
Lycoris chinensis $(2 n=16,6 \mathrm{M}+10 \mathrm{~T})$ had $10 \mathrm{FISH}$ signals of $45 \mathrm{~S}$ and $12 \mathrm{FISH}$ signals of $5 \mathrm{~S}$ rDNAs. Each T-type chromosome had one $45 \mathrm{~S}$ rDNA FISH signal at the end of its p-arm (T1p-T10p). Each 45S rDNA locus on these T-type chromosomes, except for T10, was colocalized with a 5S rDNA locus. The remaining 5S rDNA FISH signals included one near the centromere of M3 and two conspicuous signals at the subtelomeric regions of T7q and T8q (Fig. 3).

A-KARYotype. The number and positions of $5 \mathrm{~S}$ and $45 \mathrm{~S}$ rDNA loci varied among the species with an A -karyotype $(2 n=$ 22, 22A) (Table 1; Fig. 4).

Lycoris sprengeri and its curly leaved variant (accession LSM) had the same pattern of rDNA FISH signals with two $45 \mathrm{~S}$ and four 5S rDNA FISH signals (Fig. 4A). Lycoris radiata var. pumila had four 45S and four 5S rDNA FISH signals (Fig. 4B), whereas its variant collected from Ma-Tzu, L. rosea, and L. haywardii had three $45 \mathrm{~S}$ and four $5 \mathrm{~S}$ rDNA FISH signals (Fig. 4C). The FISH signals of the $45 \mathrm{~S}$ rDNAs as well as the $5 \mathrm{~S}$ rDNAs were heterozygous in these three genomes (Fig. 4C). The three 45S rDNA signals were at the end of chromosomes A1p, A2p, and A3p. There were four 5S rDNA FISH signals:

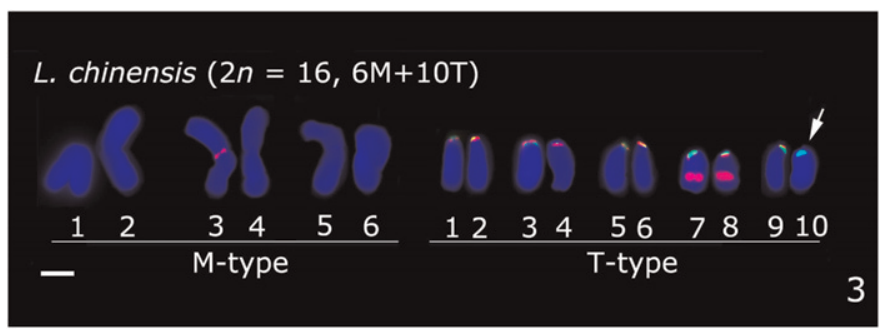

Fig. 3. rDNA fluorescent in situ hybridization signals on the chromosome complement of Lycoris chinensis $(2 n=16,6 \mathrm{M}+10 \mathrm{~T})$. The signals of $5 \mathrm{~S}$ rDNA (red) overlapped with the signals of 45S rDNA (green) at the end of the p-arm of T-type chromosomes, except for T10 (arrow); scale bar $=10 \mu \mathrm{m}$.

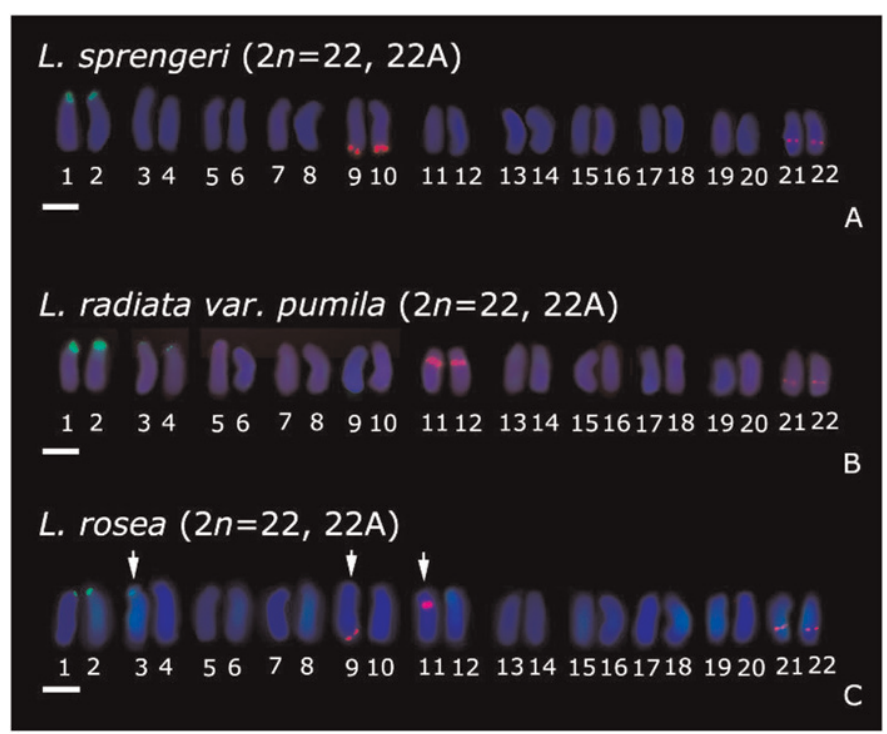

Fig. 4. rDNA fluorescent in situ hybridization (FISH) signals on the chromosome complements of three representative A-type species $(2 n=22,22 \mathrm{~A})$ : (A) Lycoris sprengeri, (B) Lycoris radiata var. pumila, and (C) Lycoris rosea. Chromosomes (blue) were hybridized with a probe of 5S rDNAs (red) and a probe of $45 \mathrm{~S}$ rDNAs (green). Arrows indicate the heterozygous rDNA FISH signals on homologous chromosomes; scale bar $=10 \mu \mathrm{m}$. one at the distal end of $\mathrm{A} 9 \mathrm{q}$, one in the proximal region of A11q, and two at the subtelomeric regions of A21q and A22q (Fig. 4C).

MT-A KARYOTYPE. Lycoris albiflora, L. ×houdyshelii, and three artificial dikaryotype hybrids were the studied taxa with an MT-A dikaryotype (Table 1).

Lycoris albiflora $(2 n=17,5 \mathrm{M}+1 \mathrm{~T}+11 \mathrm{~A})$, which is considered a hybrid between $L$. traubii $(2 n=12,10 \mathrm{M}+2 \mathrm{~T})$ and L. radiata var. radiata $(2 n=3 \mathrm{x}=33,33 \mathrm{~A})$ (Kurita, 1987a), had four $45 \mathrm{~S}$ and six $5 \mathrm{~S}$ rDNA FISH signals on chromosomes (Table 1; Fig. 5A). On T1p, the FISH signals of $45 \mathrm{~S}$ rDNAs overlapped with that of the $5 \mathrm{~S}$ rDNAs (Fig. 5A).

Lycoris $\times$ houdyshelii $(2 n=30,3 \mathrm{M}+5 \mathrm{~T}+22 \mathrm{~A})$, a hybrid between L. longituba $(2 n=16,6 \mathrm{M}+10 \mathrm{~T})$ and an unknown donor with $2 n=22 \mathrm{~A}$ (Kurita, 1987a), had 12 FISH signals of $45 \mathrm{~S}$ rDNAs and 14 FISH signals of 5S rDNAs (Fig. 5B; Table 1). Each T-type chromosome, except for T4, had 45S rDNA FISH signals overlapped with $5 \mathrm{~S}$ rDNA FISH signals at the distal end of its p-arm (T1p-T3p, T5p). On chromosome T4, one conspicuous FISH signal of $45 \mathrm{~S}$ rDNAs was present at the end of its p-arm and FISH signals of $45 \mathrm{~S}$ and $5 \mathrm{~S}$ rDNAs were overlapped at its q-arm (Fig. 5B).

An artificial hybrid $(2 n=18,4 \mathrm{M}+3 \mathrm{~T}+11 \mathrm{~A})$ of $L$. aurea $(2 n=14,8 \mathrm{M}+6 \mathrm{~T}) \times L$. radiata var. pumila $(2 n=22 \mathrm{~A})$ had four 45S rDNA FISH signals and eight to nine $5 \mathrm{~S}$ rDNA FISH signals (Fig. 6A). Like in L. ×houdyshelii, each T-type chromosome had $45 \mathrm{~S}$ rDNA FISH signals overlapped with $5 \mathrm{~S}$ rDNA signals at the distal end of its p-arm (T1p-T3p). It is interesting to note that one particular studied plant had an additional, although weak, $5 \mathrm{~S}$ rDNA FISH signal detected at the distal end of M4q (Fig. 6A).

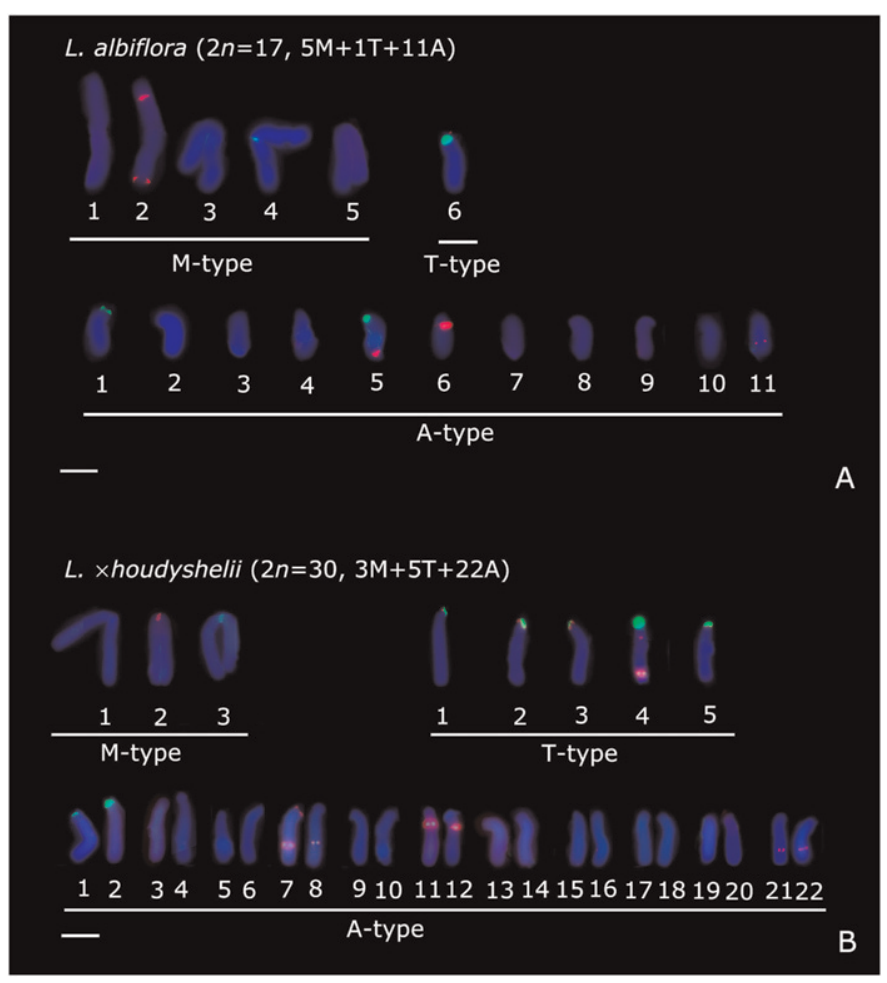

Fig. 5. rDNA fluorescent in situ hybridization signals on the chromosome complements of an MT-A dikaryotype Lycoris species and hybrids: (A) Lycoris albiflora $(2 n=17,5 \mathrm{M}+1 \mathrm{~T}+11 \mathrm{~A})$ and $(\mathbf{B})$ Lycoris $\times$ houdyshelii $(2 n=30,3 \mathrm{M}+5 \mathrm{~T}+22 \mathrm{~A})$. Chromosomes (blue) were hybridized with a probe of $5 \mathrm{~S}$ rDNAs (red) and a probe of $45 \mathrm{~S}$ rDNAs (green); scale bar $=10 \mu \mathrm{m}$. 


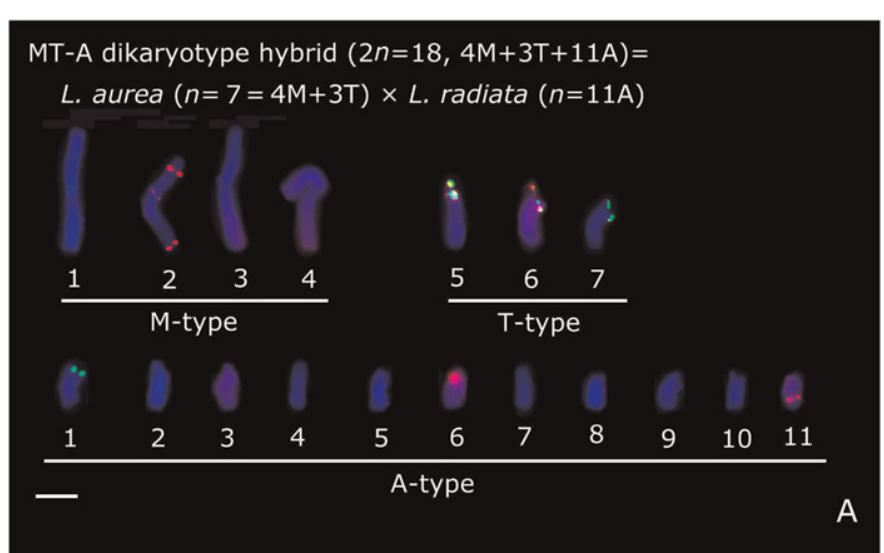

MT-A dikaryotype hybrid $(2 n=19,3 M+5 T+11 \mathrm{~A})=$

L. chinensis $(n=8=3 M+5 T) \times L$. rosea $(n=11 \mathrm{~A})$

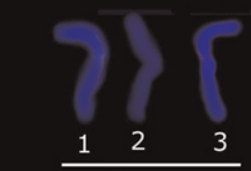

M-type

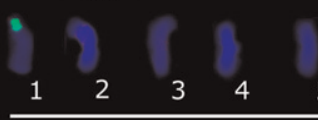

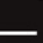

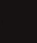

A-type

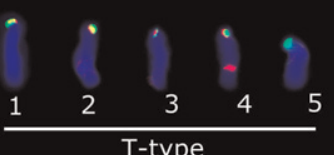

T-type

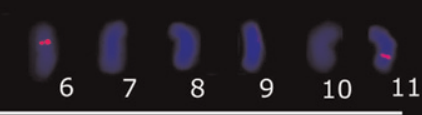

MT-A dikaryotype hybrid $(2 n=19,3 M+5 T+11 \mathrm{~A})=$

L. longituba $(n=8=3 M+5 T) \times$ L. radiata var. pumila $(n=11 \mathrm{~A})$

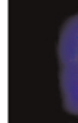

(2)

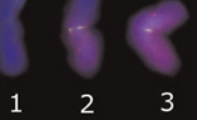

M-type

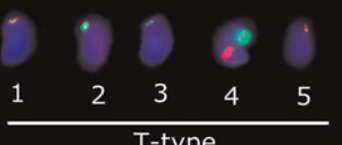

T-type

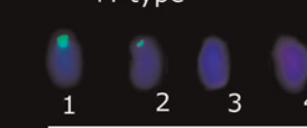

-

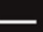

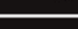

$4 \quad 5$

A-type

Fig. 6. rDNA fluorescent in situ hybridization signals on the chromosome complements of three artificial MT-A dikaryotype hybrids: (A) Lycoris aurea $(n=7,4 \mathrm{M}+3 \mathrm{~T}) \times$ Lycoris radiata var. pumila $(n=11 \mathrm{~A})=(2 n=18,4 \mathrm{M}+3 \mathrm{~T}$ $+11 \mathrm{~A}),(\mathbf{B})$ Lycoris chinensis $(n=8,3 \mathrm{M}+5 \mathrm{~T}) \times \operatorname{L}$. rosea $(n=11 \mathrm{~A})=(2 n=19$, $3 \mathrm{M}+5 \mathrm{~T}+11 \mathrm{~A})$, and $(\mathbf{C})$ Lycoris longituba $(n=8,3 \mathrm{M}+5 \mathrm{~T}) \times$ L. radiata var. pumila $(n=11 \mathrm{~A})=(2 n=19,3 \mathrm{M}+5 \mathrm{~T}+11 \mathrm{~A})$. Chromosomes (blue) were hybridized with a probe of $5 \mathrm{~S}$ rDNAs (red) and a probe of $45 \mathrm{~S}$ rDNAs (green); scale bar $=10 \mu \mathrm{m}$.

An artificial dikaryotype hybrid $(2 n=19,3 \mathrm{M}+5 \mathrm{~T}+11 \mathrm{~A})$ of L. chinensis $(2 n=6 \mathrm{M}+10 \mathrm{~T}) \times L$. rosea $(2 n=22 \mathrm{~A})$ had six $45 \mathrm{~S}$ rDNA and seven 5S rDNA FISH signals (Fig. 6B). FISH signals of $45 \mathrm{~S}$ rDNAs were located at the distal end of the p-arm of all T-type chromosomes (T1p-T5p), four of which (T1p-T4p) overlapped with $5 \mathrm{~S}$ rDNA FISH signals. T4q possessed an additional 5S rDNA locus at its intercalary segment (Fig. 6B).

Another artificial dikaryotype hybrid $(2 n=19,3 \mathrm{M}+5 \mathrm{~T}+$ $11 \mathrm{~A})$ of $L$. longituba $(2 n=16,6 \mathrm{M}+10 \mathrm{~T}) \times L$. radiata var. pumila $(2 n=22 \mathrm{~A})$ had nine $45 \mathrm{~S}$ and $105 \mathrm{~S}$ rDNA FISH signals. Seven of the 45S rDNA FISH signals overlapped with 5S rDNA FISH signals on two M-type and five T-type chromosomes (Fig.
6C). T4q possessed a conspicuous 5S rDNA FISH signal at its intercalary segment. Two FISH signals of $45 \mathrm{~S}$ rDNAs and two of 5S rDNAs were located at four A-type chromosomes (Fig. 6C).

\section{Discussion}

Studies based on rDNA FISH have revealed the variations in the number, size, and distribution pattern of both the $45 \mathrm{~S}$ and $5 \mathrm{~S}$ rDNA loci among Lycoris taxa. These variations are likely the consequences of frequent events of interspecific hybridization and chromosome rearrangements in Lycoris. Similar observations have been reported in a few other plants, including Oryza species, Nicotiana species, and Aegilops species. In allotetraploid Oryza species, the number of the 45S rDNA loci was not necessarily equal to the sum of their progenitors (Chung et al., 2008). Diploidization in the allopolyploid Nicotiana section Repandae (Solanaceae) was associated with the loss of a 5S rDNA locus (review by Clarkson et al., 2005). In the genus Aegilops, significant variations in the chromosomal position and number of rDNA loci were associated with chromosome repatterning, which is a known process of speciation in Triticum species (review by Raskina et al., 2004).

Various genetic alterations have been reported in some interspecific hybrids, including gene elimination, amplification, and transposition (Comai, 2000; Comai et al., 2003). In this study, rDNA FISH analyses indicated several alterations in the number and positions of the rDNA loci in both natural and artificial interspecific hybrids. Lycoris rosea $(2 n=22 \mathrm{~A}$; Fig. 4C) and L. haywardii (data not shown) are considered as two derivatives of a natural hybrid between L. sprengeri $(2 n=22 \mathrm{~A})$ and $L$. radiata var. pumila $(2 n=22 \mathrm{~A})$ (Hsu et al., 1994); their phylogenetic relationships have been suggested by molecular analyses (Hori et al., 2006; Shi et al., 2006). Our rDNA FISH results also supported such phylogenetic relationships among these ancestral A-karyotype species and their resulting hybrids. In $L$. rosea $(2 n=22 \mathrm{~A})$ and $L$. haywardii (data not shown), the numbers and positions of both $45 \mathrm{~S}$ and $5 \mathrm{~S}$ rDNA FISH signals (Fig. 4C) are equal to the combination of those in L. sprengeri (Fig. 4A) and L. radiata var. pumila (Fig. 4B). The same conclusion was drawn by comparing the patterns of rDNA FISH on the chromosomes of L. radiata var. pumila (Fig. 4B) and those of the A-complement in an artificial hybrid $(2 n=18$, $4 \mathrm{M}+3 \mathrm{~T}+11 \mathrm{~A})$ of $L$. aurea $(2 n=14,8 \mathrm{M}+6 \mathrm{~T}) \times$ L. radiata var. pumila $(2 n=22 \mathrm{~A})(6 \mathrm{~A})$. Lycoris traubii $(2 n=12,10 \mathrm{M}+2 \mathrm{~T})$ and $L$. radiata var. pumila (Fig. 4B) were considered as the respective MT- and A-complement donors of $L$. albiflora $(2 n=$ $17,5 \mathrm{M}+1 \mathrm{~T}+11 \mathrm{~A}$; Kurita, 1987a); therefore, the number and position of the $45 \mathrm{~S}$ and $5 \mathrm{~S}$ rDNA loci in L. traubii can be deduced from those of the MT-complement in L. albiflora (Table 1; Fig. 5A). However, the rDNA FISH signals in the Acomplement of L. albiflora (Fig. 5A) differed from those of L. radiata var. pumila (Fig. 4B). In other words, new rDNA loci in the A-complements of L. albiflora arising after the hybrid formation. Lycoris longituba $(n=8,3 \mathrm{M}+5 \mathrm{~T})$ is the MT complement donor of both the natural hybrid $L$. $\times$ houdyshelii $(2 n=30$, $3 \mathrm{M}+5 \mathrm{~T}+22 \mathrm{~A}$, Fig. 5B) and the artificial dikaryotype hybrid L. longituba $\times$ L. radiata var. pumila $(2 n=19,3 \mathrm{M}+5 \mathrm{~T}+11 \mathrm{~A}$; Fig. 6C); however, the rDNA FISH patterns on the MTcomplements of these two hybrids were not identical. It appears that rDNA repeats had undergone a transposition and that the telomeric sequences were amplified during the formation of $L \times$ houdyshelii (Figs. 5B and 6C). 
Lycoris radiata is a species complex; the diploid populations belong to L. radiata var. pumila Grey (Hsu et al., 1994), whereas the sterile triploid populations belong to $L$. radiata var. radiata (Kurita, 1987b). The L. radiata populations found in China were reported to display variations in the chromosome numbers and karyotypes among populations and within a population (Zhou et al., 2007). In this study, L. radiata var. pumila and that variant that we collected in Ma-Tzu showed different patterns of rDNA FISH (Fig. 4B-C). That variant of $L$. radiata var. pumila as well as $L$. rosea and L. haywardii were detected to have heterozygous rDNA loci (Fig. 4C). Similar heterozygous rDNA loci were also reported in $L$. sanguinea var. koreana (Lee and Kim, 2004).

Robertsonian fusion and fission mechanism are two of the most important types of karyotype evolution in animals but relatively uncommon in higher plants (Jones, 1998). A centromeric fusion of two acrocentric chromosomes forms a metacentric chromosome and a centromeric fission of one metacentric chromosome produces two telocentric chromosomes. Because these types of chromosomal rearrangements involve the whole chromosome arms, the chromosome number and morphology are changed, whereas the number of major chromosome arms remains constant. Plants of the Amaryllidaceae have been reported to change karyotypes by whole arm rearrangement (Jones, 1998). Numerous studies have provided a considerable amount of information regarding the phylogeny and karyotype evolution of the genus Lycoris. However, the knowledge of the ancestral karyotypes based on a reliable method for chromosome identification was still lacking. Previous reports indicated that the karyotypic variations in the genus Lycoris are consequences of either fusion or fission. Inariyama (1951) proposed that the ancestral karyotype of Lycoris species was $2 n=22 \mathrm{~A}$, and that a species with $2 n=12$ $(10 \mathrm{M}+2 \mathrm{~T})$ resulted from centric fusion. The phylogenetic analysis of chloroplast genome sequences also suggests that L. radiata var. pumila $(2 n=22 \mathrm{~A})$ is a possible ancestral taxon of the genus Lycoris (Hori et al., 2006). In contrary, Kurita $(1986,1988)$ suggested that the species with M-type chromosomes were the most ancestral, and the T- type chromosome derived from the M-type chromosomes through Robertsonian fission, then T-type chromosome converted into the A-type chromosome by pericentric inversion.

The locations of $45 \mathrm{~S}$ rDNA loci have been detected at the centromeric ends of all T-type chromosomes, the distal regions of the short arm of some A-type chromosomes, and the distal end of each arm of M-type chromosomes in some interspecific hybrids of Lycoris (Ogawa et al., 2006). Our study showed that almost all T-type chromosomes (but not the A-type chromosomes) had 45S rDNA signals, 5S rDNA signals, and DAPIpositive bands overlapped at the end of their p-arms (Figs. 2, 3, 5 , and 6). As a result of the limited resolution of FISH and the highly condensed feature of the metaphase chromosomes, overlap of $45 \mathrm{~S}$ rDNA and $5 \mathrm{~S}$ rDNA FISH signals does not necessarily mean that they are at the same locus. Rather, it is more likely that they are in close proximity. We observed the signals of $5 \mathrm{~S}$ and $45 \mathrm{~S}$ rDNA FISH overlapped even in interphase nuclei (data not shown), suggesting the intermixing of $5 \mathrm{~S}$ and $45 \mathrm{~S}$ rDNAs in Lycoris. In higher eukaryotes, the 45S rDNA and 5S rDNA loci are transcribed by different RNA polymerases and usually located in different positions of chromosomes (Srivastava and Schlessinger, 1991). Colocalization of $45 \mathrm{~S}$ and $5 \mathrm{~S}$ rDNA loci has commonly been reported in animals (Dobigny et al., 2003). Although it has also been reported in some plants such as Artemisia species (Garcia et al., 2007) and plants in Chrysantheminae (Abd El-Twab and Kondo, 2006, 2007), it was unknown in Lycoris previously. The insertion of $5 \mathrm{~S}$ rDNA into $45 \mathrm{~S}$ rDNA is thought to be accidental with unknown significance, which may be mediated by transposons or retrotransposons (Drouin and Moniz de Sa, 1995). Like in Artemisia species (Garcia et al., 2007), 5S and 45S rDNA loci colocalized with DAPI-positive bands were observed on the T-type chromosomes of Lycoris species. On the other hand, fewer heterochromatin or rDNA FISH signals were detected in the pericentric region of the M-type chromosomes (Figs. 2-6). The different distribution patterns of rDNAs and heterochromatin on T-type chromosomes and M-type chromosomes shown in this study indicate that T-type chromosomes may be derived from M-type chromosomes by centromeric fission in Lycoris species. During this process, several repetitive sequences, including telomeric repeats, $45 \mathrm{~S}$ rDNAs, and 5S rDNAs, were amplified and added to the broken ends to stabilize those new formed T-type chromosomes (Jones, 1998).

In conclusion, rDNA FISH and DAPI staining are reliable and efficient methods for chromosome identification in Lycoris taxa. The rDNA FISH results presented here reveal the variations of rDNA loci among different karyotypes in the genus Lycoris. These variations reflect the frequent events of chromosome rearrangements and interspecific hybridization in Lycoris species. However, karyotyping with rDNA loci is not sufficient to determine the corresponding relationships among the A-, T-, and M-type chromosomes in Lycoris species. To further clarify the process of karyotype changes in the genus Lycoris, a set of chromosomal arm-specific landmarks are required to implement a FISH-based identification system for chromosome arms.

\section{Literature Cited}

Abd El-Twab, M.H. and K. Kondo. 2006. FISH physical mapping of 5S, 45S and Arabidopsis-type telomere sequence repeats in Chrysanthemun zawadskii showing intra-chromosomal variation and complexity in nature. Chromosome Bot. 1:1-5.

Abd El-Twab, M.H. and K. Kondo. 2007. FISH physical mapping of $5 \mathrm{~S} \mathrm{rDNA}$ and telomere sequence repeats identified a peculiar chromosome mapping and mutation in Leucanthemella linearis and Nipponanthemum nipponicum in Chrysanthemum sensu lato. Chromosome Bot. 2:11-17.

Ansari, H.A., N.W. Ellison, S.M. Reader, E.D. Badaeva, B. Friebe, T.E. Miller, and W.M. Williams. 1999. Molecular cytogenetic organization of $5 \mathrm{~S}$ and $18 \mathrm{~S}-26 \mathrm{~S}$ rDNA loci in white clover (Trifolium repens L.) and related species. Ann. Bot. (Lond.) 83:199-206. Badaeva, E.D., B. Friebe, and B.S. Gill. 1996. Genome differentiation in Aegilops. 1. Distribution of highly repetitive DNA sequences on chromosomes of diploid species. Genome 39:293-306.

Cabrero, J. and J.P.M. Camacho. 2008. Location and expression of ribosomal RNA genes in grasshoppers: Abundance of silent and cryptic loci. Chromosome Res. 16:595-607.

Castilho, A. and J.S. Heslop-Harrison. 1995. Physical mapping of 5S and 18S-25S rDNA and repetitive DNA sequences in Aegilops umbellulata. Genome 38:91-96.

Chung, M.C., Y.I. Lee, Y.Y. Cheng, Y.J. Chou, and C.F. Lu. 2008. Chromosomal polymorphism of ribosomal genes in the genus Oryza. Theor. Appl. Genet. 116:745-753.

Chung, M.C., C.N. Ning, and H.K. Wu. 1993. Localization of ribosomal RNA genes on rice chromosomes. Bot. Bul. Acad. Sinica $34: 43-55$. 
Chung, M.G. 1999. Notes on allozyme variation in Lycoris radiata (Amaryllidaceae) from Korea. Bot. Bul. Acad. Sinica 40:227-230.

Clarkson, J.J., K.Y. Lim, A. Kovarik, M.W. Chase, S. Knapp, and A.R. Leitch. 2005. Long-term genome diploidization in allopolyploid Nicotiana section Repandae (Solanaceae). New Phytol. 168:241252.

Comai, L. 2000. Genetic and epigenetic interactions in allopolyploid plants. Plant Mol. Biol. 43:387-399.

Comai, L., A.P. Tyagi, and M.A. Lysak. 2003. FISH analysis of meiosis in Arabidopsis allopolyploids. Chromosome Res. 11:217226.

Dobigny, G., C. Ozouf-Costaz, C. Bonillo, and V. Volobouev. 2003. Evolution of rRNA gene clusters and telomeric repeats during explosive genome repatterning in Taterillus $\times$ (Rodentia, Gerbillinae). Cytogenet. Genome Res. 103:94-103.

Drouin, G. and M. Moniz de Sa. 1995. The concerted evolution of $5 \mathrm{~S}$ ribosomal genes linked to the repeat units of other multigene families. Mol. Biol. Evol. 12:481-493.

Fransz, P., S. Armstrong, C. Alonso-Blanco, T.C. Fisher, R. TorresRuiz, and G. Jones. 1998. Cytogenetics for the model system Arabidopsis thaliana. Plant J. 13:867-876.

Garcia, S., T. Garnatje, O. Hidalgo, E.D. McArthur, S. SiljakYakovlev, and J. Valles. 2007. Extensive ribosomal DNA (18S5.8S-26S and 5S) colocalization in the North American endemic sagebrushes (subgenus Tridentatae, Artemisia, Asteraceae) revealed by FISH. Plant Syst. Evol. 267:79-92.

Gerlach, W.L. and J.R. Bedbrook. 1979. Cloning and characterization of ribosomal RNA genes from wheat and barley. Nucleic Acids Res. 7:1869-1885.

Gerlach, W.L. and T.A. Dyer. 1980. Sequence organization of the repeated units in the nucleus of wheat which contain $5 \mathrm{~S}$ rRNA genes. Nucleic Acids Res. 8:4851-4865.

Hayashi, A., T. Saito, Y. Mukai, S. Kurita, and T. Hori. 2005. Genetic variations in Lycoris radiata var. radiata in Japan. Genes Genet. Syst. 80:199-212.

Hori, T., A. Hayashi, T. Sasanuma, and S. Kurita. 2006. Genetic variations in the chloroplast genome and phylogenetic clustering of Lycoris species. Genes Genet. Syst. 81:243-253.

Hsu, P.S., S. Kurita, Z.Z. Yu, and J.Z. Lin. 1994. Synopsis of the genus Lycoris (Amaryllidceae). Sida 16:301-331.

Inariyama, S. 1951. Cytological studies in the genus Lycoris (II). Sci. Rep. Tokyo Bunrika Daigaku. Sect. B 7:103-156.

Jones, K. 1998. Robertsonian fusion and centric fission in karyotype evolution of higher plants. Bot. Rev. 64:273-289.

Kao, F.I., Y.Y. Cheng, T.Y. Chow, H.H. Chen, S.M. Liu, C.H. Cheng, and M.C. Chung. 2006. An integrated map of Oryza sativa L. chromosome 5. Theor. Appl. Genet. 112:891-902.

Kim, M. and S. Lee. 1991. A taxonomical of the Korean Lycoris (Amaryllidaceae). Korean J. Plant Taxonomy 21:123-139.

Kurita, S. 1986. Variation and evolution in the karyotype of Lycoris, Amaryllidaceae. 1. General karyomorphological characteristics of the genus. Cytologia (Tokyo) 51:803-815.

Kurita, S. 1987a. Variation and evolution on the karyotype of Lycoris, Amaryllidaceae. II. Karyotype analysis of ten taxa among which seven are native in China. Cytologia (Tokyo) 52:19-40.

Kurita, S. 1987b. Variation and evolution in the karyotype of Lycoris, Amaryllidaceae. IV. Interspecific variation in the karyotype of $L$. rediata (L'Herit.) Herb. and the origin of this triploid species. Cytologia (Tokyo) 52:137-149.

Kurita, S. 1988. Variation and evolution in the karyotype of Lycoris, Amaryllidaceae. VII. Modes of karyotype alteration within species and probable trend of karyotype evolution in the genus. Cytologia (Tokyo) 53:323-335.
Kurita, S. 1989. Variation and evolution in the karyotype of Lycoris (Amaryllidaceae). V. Chromosomal variation in $L$. sanguinea Maxim. Plant Species Biol. 4:47-60.

Kurita, S. and P.S. Hsu. 1996. Hybrid complex in Lycoris. Amaryllidaceae. Amer. J. Bot. 83:207.

Kurita, S. and P.S. Hsu. 1998. Cytological patterns in the SinoJapanese flora. Hybrid complexes in Lycoris, Amaryllidaceae, p. 171-180. In: D.E. Boufford and H. Ohba (eds.). Sino-Japanese flora its characteristics and diversification. Bul. No. 37. University Museum, Univ. Tokyo, Tokyo, Japan.

Lee, B. and M. Kim. 1987. Palynological study of some Lycoris species. Korean J. Plant Taxonomy 17:147-154.

Lee, B. and M. Kim. 2000. Chromosomal localization of rDNA genes in the Korean endemic Lycoris flavescens M. Kim et S. Lee and its related species (Amaryllidaceae). Korean J. Genet. 22:1-17.

Lee, B. and M. Kim. 2004. Genome characterization of a Korean endemic species Lycoris chejuensis (Amaryllidaceae) by in situ hybridization. Korean J. Genet. 26:83-89.

Linde-Laursen, I., E. Ibsen, R. Von Bothmer, and H. Giese. 1992. Physical localization of active and inactive rRNA gene loci in Hordeum marinum ssp. gussoneanum $(4 \mathrm{x})$ by in situ hybridization. Genome 35:1032-1036.

Liu, Y. and P.S. Hsu. 1989. A study on karyotypes of the genus Lycoris. Acta Phytotaxon. Sinica 27:257-264.

Ma, B., T. Ogawa, and I. Tarumoto. 2004. Genetic segregation of allozymes in selfed progenies of diploid Lycoris species (Amaryllidaceae). Sci. Rpt. Graduate School Agr. Biol. Sci. Osaka Prefecture. Univ. 56:17-22.

McClintock, B. 1934. The relationship of a particular chromosomal element to the development of the nucleoli in Zea mays. Zeitschrift fur Zellforschung und microskopische Anatomie 21:294-328.

Nishiyama, I. 1928. Reduction division in Lycoris. Bot. Mag. Tokyo 42:509-513.

Ogawa, T., I. Tarumoto, B. Ma, M. Ueno, and T. Morikawa. 2006. A study on interspecific hybrids and selfed progeny of Lycoris by means of fluorescence in situ hybridization. Breed. Sci. 56:209212.

Pedrosa-Harand, A., C.C.S. de Almeida, M. Mosiolek, M.W. Blair, D. Schweizer, and M. Guerra. 2006. Extensive ribosomal DNA amplification during Andean common bean (Phaseolus vulgaris L.) evolution. Theor. Appl. Genet. 112:924-933.

Raskina, O., A. Belyayev, and E. Nevo. 2004. Quantum speciation in Aegilops: Molecular cytogenetic evidence from rDNA cluster variability in natural populations. Proc. Natl. Acad. Sci. USA 101: 14818-14823.

Shi, S., Y. Qsiu, E. Li, L. Wu, and C. Fu. 2006. Phylogenetic relationships and possible hybrid origin of Lycoris species (Amaryllidaceae) revealed by ITS sequences. Biochem. Genet. 44:198208.

Srivastava, A.K. and D. Schlessinger. 1991. Structure and organization of ribosomal DNA. Biochimie 73:631-638.

Suzuki, H., S. Sakurai, and Y. Matsuda. 1996. Rat rDNA spacer sequences and chromosomal segment of the genes to the extreme terminal region of chromosome 19. Cytogenet. Cell Genet. 72:1-4.

Taketa, S., G.E. Harrison, and J.S. Heslop-Harrison. 1999. Comparative physical mapping of the $5 \mathrm{~S}$ and $18 \mathrm{~S}-25 \mathrm{~S}$ rDNA in nine wild Hordeum species and cytotypes. Theor. Appl. Genet. 98:1-9.

Yuan, M.S., C.J. Wu, and C.T. Shii. 1998. Fertility and karyotype remodel in the dikaryotype hybrids Lycoris aurea $\times L$. radiata. J. Genet. Mol. Biol. 9:91-99.

Zhou, S.B., B.Q. Yu, Q. Luo, J.R. Hu, and D. Bi. 2007. Karyotypes of six populations of Lycoris radiata and discovery of the tetraploid. Acta Phytotaxon. Sinica 45:513-522. 